\title{
INTROdUCtION TO THE Fourier TRASNFORM: IMAGE PROCESSING LABORATORY EXAMPLE
}

\author{
Gabriel Thomas \\ Electrical and Computer Engineering Department, University of Manitoba \\ thomas@ee.umanitoba.ca
}

\begin{abstract}
Signal processing can be a mathematical intense subject and undergraduate students may not be able to appreciate the enormous importance on the applied side of things. Even if the instructor mentions some of the fascinating application areas, it might be difficult for students to attend a laboratory session in which they will have enough time to code a signal processing algorithm and see a big difference when processing data in the frequency domain. In this paper I describe a simple example in which students have an opportunity to develop a theoretical solution to enhance an image and with just a few lines of code run a program that will show a dramatic difference between an original image and the enhanced version. Different fundamental signal processing concepts are reinforced with the laboratory example and its application only requires a computer and signal processing developing software such as Matlab.
\end{abstract}

Keywords: Signal processing, image enhancement, Fourier transform, undergraduate laboratory.

\section{INTRODUCTION AND MOTIVATION}

The development of exciting undergraduate laboratories that can offer students firsthand experience on heavy theoretical concepts can be a challenge. Teaching a first course on signal processing can be such a case. First thing that can be discussed is what type of examples can be categorized as exciting to most students. An audio example must be of interest to a good number of students but if the adage is true an " a picture is worth a thousand words," then an image processing example may be a better one to approach. Because the example is intended to be part of a 3 hour signal processing laboratory for a first course on Signal Processing, the challenge is to design an example that would require only a few lines of code, teach some of the basics of the Fourier Transform (FT) and analyze the signal in 1D so that to avoid the discussion of the 2D FT. This paper then presents in Section 2 the example, Section 3 covers the solution and the different FT concepts targeted in this part of the laboratory and finally conclusions can be found in Section 4.

\section{DESCRIPTION AND GENERATION OF THE IMAGE EXAMPLE}

\subsection{Description of the Problem}

The problem as presented in the laboratory hand out is described next:

A camera has a series of lenses that produce images with two ghosts one to the left and another one to the right 50 pixels apart with an attenuation factor of 0.25 . You can see this by loading the image data already given in Matlab format and using the command $\operatorname{imagesc}(A)$ followed by colormap gray and axis image to see it. Let $g(x, y)$ define this bad image and $f(x, y)$ be the good image you are looking for - no ghosts. Because, as it can be seen in Figure 1, we are only concern about the errors being introduced in the $x$ direction, do the following:

Task1:

Write $g(x)$ in terms of $f(x)$ to model these errors, that is, write the mathematical model for each row of the image.

Task 2:

Take the Fourier transform on both sides so that you can end up with an expression in frequency of the form $G(w)=H(w) F(w)$.

Task 3:

Sample the expression you found for $H(w)$ from 0 to $2 \pi$ so that you can create a vector of similar length as the length of your FTs.

Task 4:

In Matlab compute $G(w) . / H(w)$ and use the inverse Fourier command ifft to see if you can eliminate the ghosts. 


\subsection{Generation of the Image}

The software to be used in this work is based on Matlab. This section then describes how from a good image, as the one shown in Figure 1, one can generate the distortion mentioned in Section 2. It is assumed that the image file is a jpeg image and is a color image as can be seen in the first command in the first lines of the following code:

A = imread('example.jpg');

$\%$ Define amount of translation

$\mathrm{T}=50$

$\%$ Define amount of attenuation

att $=0.25$;

$\%$ The following code adds the degradation

$\mathrm{A}=\operatorname{rgb} 2 \operatorname{gray}(\mathrm{A})$;

$\mathrm{A}=\operatorname{double}(\mathrm{A})$;

$\mathrm{B} 1=\operatorname{circshift}(\mathrm{A},[0-\mathrm{T}])$;

$\mathrm{B} 2=\operatorname{circshift}(\mathrm{A},[0 \mathrm{~T}])$;

$\mathrm{A}=\mathrm{A}+\mathrm{att} * \mathrm{~B} 1+\mathrm{att} * \mathrm{~B} 2$
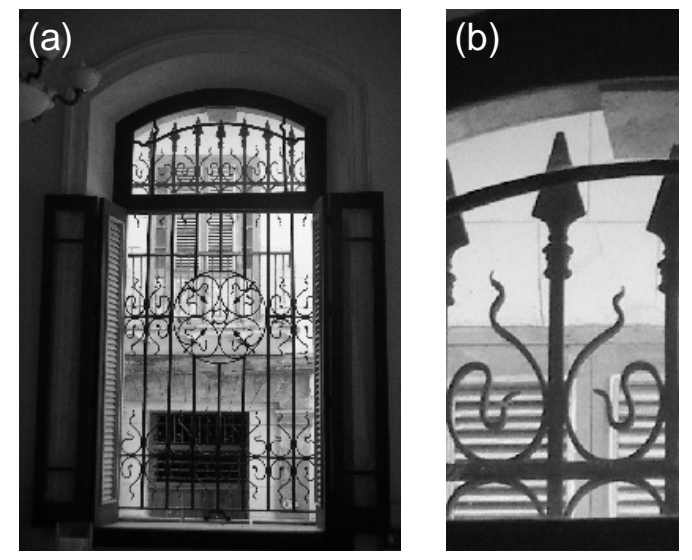

Figure 1. (a) Original image. (b) Close up of the top section.

Having run the code in this section to the image presented in Figure 1, now show the distortion to be deal with during the laboratory, please refer to Figure 2 to see the distortion.

\section{SOLUTION OF THE LABORATORY}

The tasks presented in section 2.1 will be solved here.

\subsection{Solution of Task 1, Mathematical Model of the Degradation}

Assuming that $g(x)$ is a row from Figure 2 and that its corresponding non distorted row $f(x)$ can be seen in Figure 1, then $g(x)$ in terms of $f(x)$ can be modeled as:

$$
g(x)=f(x)+\alpha f(x+T)+\alpha f(x-T)
$$

where $\alpha$ is the attenuation discussed in the description of the problem and $T$ is the number of pixels that the attenuated ghosts are positioned to the sides of the original image.
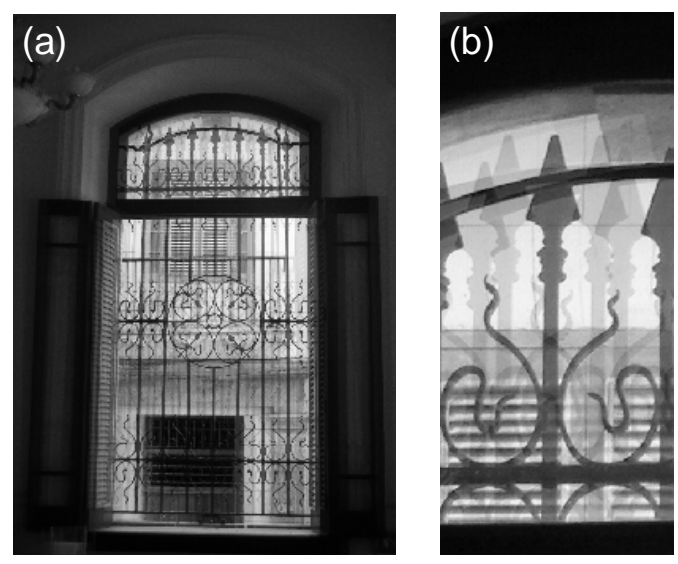

Figure 2. (a) Distorted image. (b) Close up of (a).

\subsection{Solution of Task 2, Fourier analysis of equation (1)}

As the FT of (1) is needed, the Fourier property to be used here is that of time shifting defined as [1]:

$$
x\left(t-t_{0}\right) \leftrightarrow X(w) e^{-j w t_{0}}
$$

Then applying the FT of both sides of (1) yields:

$$
G(w)=F(w)+\alpha F(w) e^{-j w T}+\alpha F(w) e^{j w T}
$$

Equation (3) can be further simplified as:

$$
G(w)=F(w)[1+2 \alpha \cos (w T)]
$$

where $G(w)=H(w) F(w)$ and thus $H(w)$ is the term in brackets in (4):

$$
H(w)=1+2 \alpha \cos (w T)
$$




\subsection{Solution of Task 3, sampling of equation $H(w)$}

This is the beginning of the discrete part of the FT analysis. Thus, the following program is the starting point for the solution that students need to implement in Matlab. It will be assumed that students have loaded the image as shown in Figure 2 and it is loaded in Matlab as matrix A.

$\%$ Identify the size of the image

$[\mathrm{s} 1 \mathrm{~s} 2]=\operatorname{size}(\mathrm{A})$;

$\%$ Define amount of translation

$\mathrm{T}=50$

$\%$ Define amount of attenuation

$\mathrm{att}=0.25$;

$\%$ Define $\mathrm{H}(\mathrm{w})$

$\mathrm{H}=\operatorname{zeros}(\mathrm{s} 1, \mathrm{~s} 2)$;

$\%$ Create frequency samples

$\mathrm{u}=$ linspace $(0,1, \mathrm{~s} 2)$;

$\mathrm{H} 1=1+2 * \operatorname{att} * \cos \left(2 * \mathrm{pi}^{*} \mathrm{~T} * \mathrm{u}\right)$;

\subsection{Solution of Task 4, Compute $G(w) / H(w)$ and use the Inverse FT}

Because images can be very big, encourage 2D implementation even though the solution is in 1D. Thus the following is the implementation of $H(w)$ as a matrix consisting on 1D filters. This part of the laboratory can be used to emphasize some Matlab characteristics such as avoiding for loops, notice how the below code starts with one simple loop that does not involves the FT in each iteration and it is just used to form the inverse filter. Students can compare this result with the implementation of the solution in 1D completely.

$$
\begin{aligned}
& \text { for } \mathrm{i}=1: \mathrm{s} 1 \\
& \mathrm{H}(\mathrm{i},:)=\mathrm{H} 1 \\
& \text { end } \\
& \text { Azf }=\mathrm{fft} 2(\mathrm{~A}) \text {; } \\
& \% \text { Do the inverse filtering; } \\
& \text { soluf = Azf./H; }
\end{aligned}
$$

$\%$ Do the inverse FT;

solur $=$ ifft2(soluf);
$\%$ Show the results;

figure

imagesc(real(solur))

colormap(gray(256))

The results are shown in Figure 3. Part (a) shows the difference between the original image and the image enhanced by using the code proposed in Section 3.4 just for illustration purposes. Students are not given the original image and cannot generate this image.
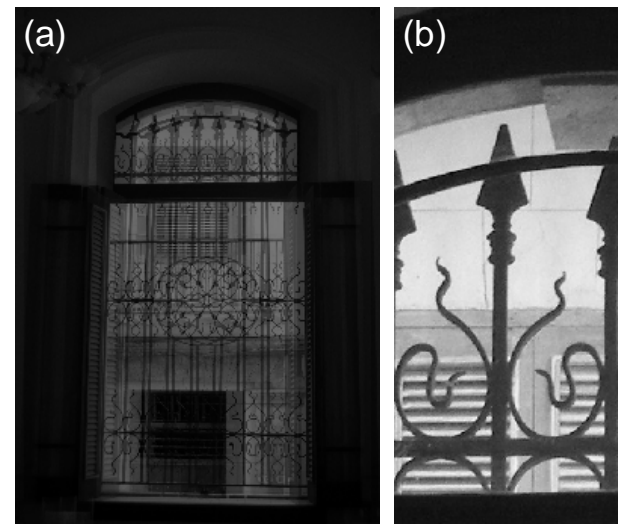

Figure 3. (a) Absolute value of the difference between the original and enhanced images. (b) Close up of enhanced image obtained by approach implemented in Section 3.

\section{FURTHER DISCUSSION}

Notice how the solution was implemented by an inverse filter so that a nice image can be simply estimated by:

$$
\hat{F}(w)=\frac{G(w)}{H(w)}
$$

Usually inverse filtering is strongly discouraged and students can be given further examples so that to emphasize that albeit theoretically accurate, implementation of inverse filtering as defined in (6) should be reconsider. Let generate the same example as computed in Section 2.2 for three different values of $\alpha$ and enhanced the images with direct inverse filtering designed for these three different values. Figure 4 shows three different cases besides the case for $\alpha=0.25$ shown already in Figure 2 (b). 

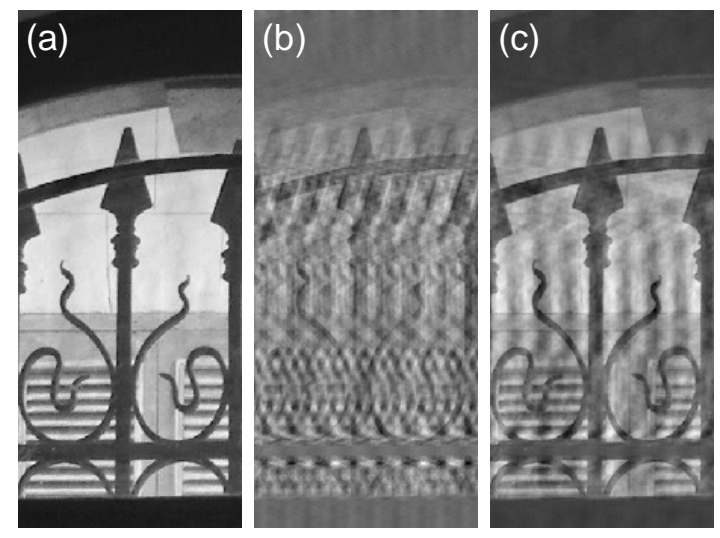

Fig. 4. (a) Enhanced image for cases $\alpha=0.45$, (b) $\alpha=0.5$, and (c) $\alpha=0.55$.

Students can be encouraged to realize that from all these examples one can conclude that good results can be obtained for values of $T$ where equation (6) does not have values very close to zero since these inversions are the ones that caused trouble. Even for blind deconvolution techniques such as the one proposed in [2], the inverse of $H(w)$ is subject to exist and be absolutely summable, that is $\sum_{w=-\infty}^{\infty}|H(w)|<\infty$.

At this particular point one can offer students a very powerful tool to enhance the cases presented in Figure 4 (b) and (c). Without a complete theoretical derivation of Wiener filtering as for example presented in [3], one can encourage to estimate the enhanced images following:

$$
\hat{F}(w)=G(w) \frac{H(w)}{H(w)^{2}+k}
$$

and explain that $k$ is a parameter to be found. Comments can be made that in case that inverse filtering does indeed yield excellent results such as the ones shown in Figure 3 (b) and Figure 4 (a), for those cases choosing a value of $k=0$ in (7) results in direct inverse filtering. Figure 5 shows the enhanced versions of all the figures presented in Figure 4 using $k=0.001$. Note should be made that even for the case where inverse filtering is not needed Figure 4(a), Wiener filtering generates a satisfactory result. All these extra examples just require the following modification of the filter in Matlab just after the last line of the code presented in Section 3.3:

$$
\begin{aligned}
& \mathrm{K}=0.001 ; \% \text { Define the parameter } \mathrm{K} \text { in the Weiner filter } \\
& \mathrm{H} 1=\mathrm{H} 1 . /\left(\mathrm{H} 1 .{ }^{\wedge} 2+\mathrm{K}\right)
\end{aligned}
$$
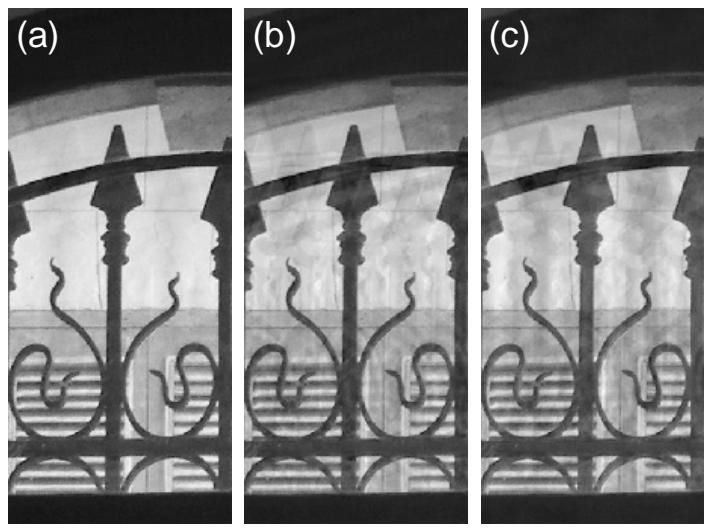

Fig. 5. Enhance images using Weiner filtering corresponding to the examples shown in Figure 4.

\section{CONCLUSIONS}

A simple example that can be part of a laboratory in an undergraduate Signal Processing course was presented. Continuous and discrete Fourier transforms concepts are evaluated and image enhancement is accomplished in which students can see the great results that can be obtained by processing an image in the frequency domain. One can even challenge them to create similar results using Photoshop. Additionally, deconvolution via Wiener filtering is presented to the students and this can offer them a tool that can be very useful in subsequent projects.

\section{References}

[1] B. P. Lathi, Linear Systems and Signals, Second Edition, Oxford University Press, New York, 2004 (2nd ed.), 992 pp. \{ISBN: 13: 978-0-19-515833-5\}

[2] D. Kundur, and D. Hatzinakos, "A novel blind deconvolution scheme for image restoration using recursive filtering," IEEE Transactions on Signal Processing, Vol.46, No.2, pp. 375-390, Feb 1998.

[3] R. C. Gonzalez and R. E. Woods, Digital Image Processing, Upper Saddle River, New Jersey, Prentice Hall, 2002 (2nd ed.), 794 pp. \{ISBN: 0-201-18075-8\}

\% Define Wiener Filter 